\title{
Gastrointestinal Stromal Tumors: Retrospective Analysis of 21 Cases
}

\author{
Tuba Atak ${ }^{1}, \odot$ Turgut Tunç Eren2 ${ }^{2}, \odot$ Büşra Burcu ${ }^{3}, \odot$ Jülide Sağıroğlu ${ }^{2}, \odot$ Ibrahim Ali Özemir ${ }^{2}$, \\ Orhan Alimoğlu² \\ ${ }^{1}$ Department of General Surgery, Cekirge State Hospital, Bursa, Turkey \\ ${ }^{2}$ Department of General Surgery, Istanbul Medeniyet University, Istanbul, Turkey \\ ${ }^{3}$ Department of General Surgery, Camlica Erdem Hospital, Istanbul, Turkey
}

\begin{abstract}
Introduction: Gastrointestinal stromal tumors (GIST) are the most common mesenchymal tumors of the digestive tract. The aim of this study was to evaluate 21 patients operated for GIST.

Methods: We retrospectively analyzed medical and surgical records of 21 patients diagnosed with GIST who were operated between 2008 and 2014 in our clinic. Gastrointestinal stromal tumors localizations, applied surgical methods, histopathological examination results, postoperative medical treatments, and follow-up results were examined.

Results: The study group consisted of 21 patients (11 males and 10 females) with a mean age of 69.29 (range, 39-89 years). Sixteen tumors were located in the stomach, 3 in the small intestine, 1 in the rectum, and 1 in the mesentery of the jejunum. The symptoms were not typical, and they depended on the localization and size of the tumor. The mean tumor size was $6.8 \mathrm{~cm}$ (range, 1.8-20 cm). Positivity rates for CD117 and CD34 expression determined by immunohistochemical methods were $95.2 \%$, and $66.7 \%$, respectively. One patient underwent laparoscopic resection, and other patients were treated via open surgery. Lymph node metastasis was observed in the patient with rectal tumor. Postoperatively, imatinib therapy was administered in 5 patients with high-risk tumors. Mortality was observed in 2 patients. Four patients were excluded from the follow-up because they could not be reached by phone. The mean follow-up period was $29.59 \pm 15.4$ months. No recurrences or metastases have been detected.

Discussion and Conclusion: Gastrointestinal stromal tumors represent $0.1 \%-1 \%$ of all gastrointestinal malignancies. The most common location is the stomach. The goal of surgery is complete gross resection with an intact pseudocapsule and negative microscopic margins.

Keywords: Gastrointestinal system; stromal tumor; surgery.
\end{abstract}

G astrointestinal stromal tumors (GISTs) are the most frequently seen mesenchymal tumors of the gastrointestinal tract, and they are frequently observed in the stomach ${ }^{[1]}$. The digestive system originates from the precursors of the interstitial cells of Cajal that regulate peristalsis. In a immunohistochemical study, C-kit (CD 117) and CD34 were positive in $95 \%$ and $60 \%-70 \%$ of cases, respectively. It is most often seen between the $4^{\text {th }}$ and 7 th decades of life. Both sexes are affected at similar rates ${ }^{[2,3]}$. Depending on the tumor size and localization, patients may present with bleeding, pain, loss of appetite, swallowing difficulty, obstruction, and perforation. A surgical intervention is the 
primary treatment. Since the lymph node involvement is rarely seen, routine lymphadenectomy is not recommended unless macroscopic involvement is observed. In patients with widespread metastases, imatinib therapy should be preferred for patients who cannot undergo total excision due to local progression, and patients whose general condition is not suitable for surgery. In this article, it is aimed to present 21 GIST cases operated between August 2008 and December 2013.

\section{Materials and Methods}

In this study, 21 patients who underwent surgical treatment and were diagnosed with GIST in Istanbul Medeniyet University General Surgery Clinic between August 2008 and December 2013 were retrospectively analyzed. Patients were evaluated according to the results of advanced pathologic examinations, in addition to their clinical and operative findings. Immunohistochemical staining characteristics, growth pattern, cellularity, cytological atypia, and numbers of mitosis were recorded. Patients were classified as low-, moderate-, and high-risk groups according to biological behavioral characteristics of the tumor after surgical resection. Locations of GIST, dimensions of the tumor, surgical methods applied, the length of stay, mortality, postoperative medical treatments, and follow-up results were examined.

\section{Results}

Of 21 patients in our study group, 11 were males and 10 were females, and the mean age was 69.29 15.29 (39-89 years). The most frequent admission complaint was abdominal pain (59.1\%). GIST was localized in the stomach $(n=16$; $76.2 \%)$, small intestine $(n=3 ; 64.8 \%)$, rectum $(n=1 ; 4.8 \%)$, and small bowel mesentery ( $n=1 ; 4.8 \%$ ) (Fig. 3). GISTs of gastric origin were situated in the corpus ( $n=14 ; 87.5 \%)$, cardia $(n=1 ; 6.25 \%)$, and fundus ( $n=1 ; 6.25 \%)$. GISTs of intestinal origin were situated in the jejunum $(n=2 ; 70.8 \%)$ and ileum $(n=1 ; 33.3 \%)$. In the entire study group, GISTs originating in the rectum $(n=1 ; 4.8 \%)$ and jejunum mesentery $(n=1 ; 4.8 \%)$ were also detected (Figs. 1,2). Open surgical ( $n=11 ; 52.3 \%)$ or laparoscopic $(n=1 ; 4.8 \%)$ wedge resections, distal subtotal gastrectomy $(n=2 ; 9.5 \%)$, total gastrectomy $(n=2 ; 9.5 \%)$, and segmental small bowel resection $(n=4 ; 119.1 \%)$ were performed. An abdominoperineal resection (Miles operation) was performed for the treatment of GIST detected in the rectum. A histopathological examination revealed bleeding in 12, necrosis in 18, and ulceration in 5 patients. The growth pattern was expansive in $18(85.7 \%)$, the infiltrative type in $2(9.5 \%)$, and the mixed type in $1(4.8 \%)$ patient. The mean tumor diameter was $6.8 \mathrm{~cm}$ (range, 1.8-20

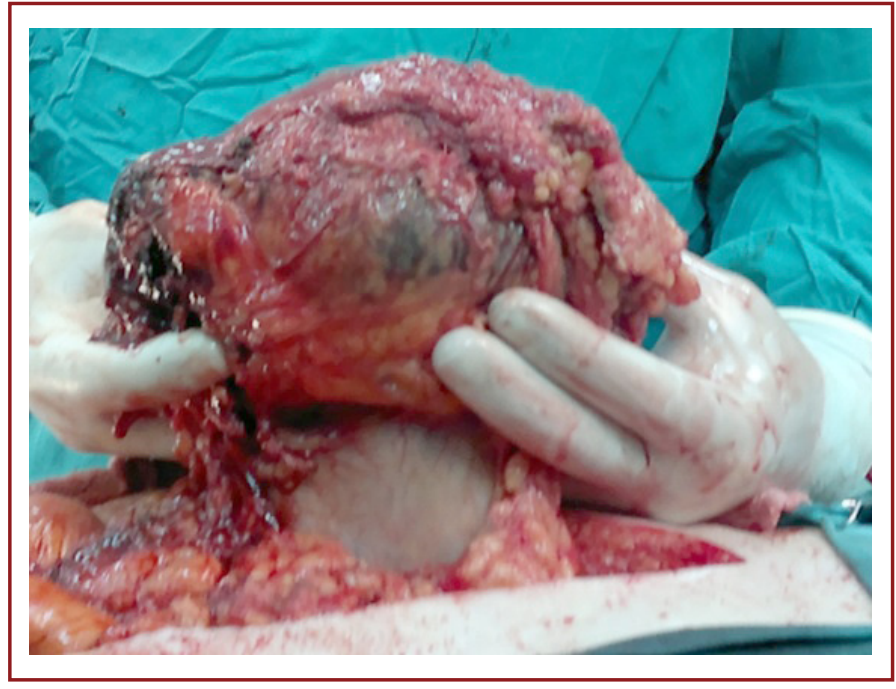

Figure 1. GIST measuring $20 \times 20 \mathrm{~cm}$ at the greater curvature of the stomach.

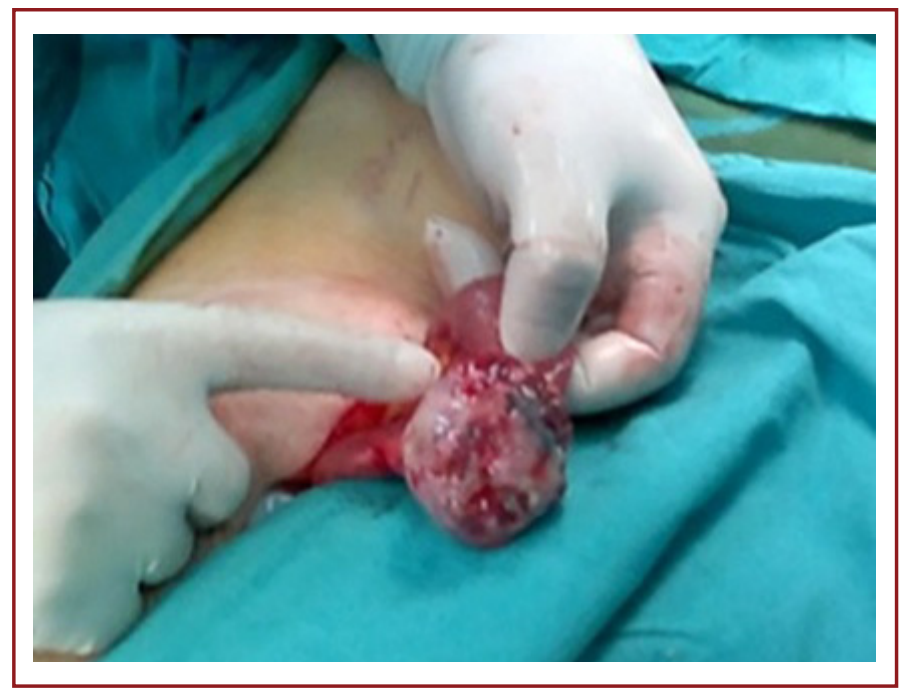

Figure 2. GIST measuring $3 \times 2 \mathrm{~cm}$ on the small intestine.

$\mathrm{cm})$. Immunohistochemical examination revealed positive expressions of CD117 in 20 (95.2\%) and CD34 expression in $14(66.7 \%)$ cases. The patients were in the low- $(n=10$; $47.6 \%)$, moderate- $(n=7 ; 33.3 \%)$ and high-risk $(n=4 ; 19.1 \%)$ categories, and none had vascular invasion. One lymph node metastasis was detected in the rectum. Cellularity was mild in $8(38.1 \%)$, moderate in $7(33.3 \%)$, and marked in $6(28.6 \%)$ patients. Cytologic atypia was mild in $12(57.2 \%)$, moderate in $6(28.6 \%)$, and severe in $3(14.2 \%)$ patients. The mitosis rate was $<5 / 50 \mathrm{BBA}$ in $18(85.7 \%), 5 / 50-10 / 50 \mathrm{BBA}$ in $1(4.7 \%)$ and $>10 / 50 \mathrm{BBA}$ in $2(9.6 \%)$ patients. The MIBI index was $<10$ in $17(80.9 \%)$ and 10 in $4(19.1 \%)$ patients.

Adjuvant imatinib therapy was started in 5 cases with established high risk. Surgical margins were evaluated as negative in all patients. Two elderly patients with comor- 
bidities exited due to cardiopulmonary complications that occurred on Days 1 and 9 postoperatively. The mean follow-up time was $27.95 \pm 15.39$ months. No recurrence or metastasis was detected in any of the patients.

\section{Discussion}

AGIST is the most frequent mesenchymal tumor of the gastrointestinal tract, accounting for $0.1 \%-3 \%$ of all gastrointestinal malignancies ${ }^{[1]}$. In the gastrointestinal system, it is seen in the stomach $(60 \%-70 \%)$, small intestine (20\%-25\%), esophagus (5\%), and colon and rectum (10\%) at respective incidence rates ${ }^{[2]}$. Less frequently, it can be also seen in the membranes of internal organs such as the mesentery, peritoneum, omentum, liver, and pancreas. Estimated frequency of occurrence is $10 \%-20 \%$ per year, with an average age of approximately 60 years (40-70 years). They are seen at similar incidence rates in both sexes ${ }^{[3,4]}$. In our series, the female-to-male ratio was equal, and the mean age was found as $69.29 \pm 15.29$ (39-89) years.

In the normal histological structure of the intestinal wall, there are interstitial Cajal cells in and around the myenteric plexus that regulate the gastrointestinal system peristalsis. GISTs are thought to be mesenchymal tumors originating from precursors of Cajal cells (5). Cajal cells contain the C-kit gene protein, which regulates intracellular events. Mutations in C-kit proto-oncogenes are involved in the pathogenesis of GISTs. In some GISTs, mutation in the platelet-derived growth factor receptor alpha (PGFRa) gene, another tyrosine kinase proto-oncogene, is detected instead of the C-kit mutation, but mutation cannot be detected in some cases.

C-kit is an immune marker of CD117 ${ }^{[6,7]}$. Most GISTs have been diagnosed with leiomyoma or leiomyosarcoma in the past ${ }^{[8]}$. Today, they are classified as C-kit or CD34-positive mesenchymal tumors ${ }^{[9]}$. In the immunohistochemical examination, $95 \%$ of C-kit (CD117), $60 \%-70 \%$ of CD34, $30 \%-$ $40 \%$ of SMA, and $5 \%$ of S-100 protein are positive. Leiomyomas or leiomyosarcomas are desmin and SMA-positively stained tumors, while CD117 and CD34 do not stain with desmin and SMA. Similarly, schwannomas are also positively stained for S-100, while CD117 and CD34 are not stained. Immunohistochemical methods aid in differential diagnosis of GIST [10]. In our series, CD117 expression was found to be positive in 20 (95.2\%) and CD34 expression in 14 cases $(66.7 \%)$ in accordance with the literature.

From an epidemiological point of view, GISTs emerge as sporadic or family-based cases. Familial GIST shows an autosomal dominant transmission [11]. In Type I neurofibromatosis, the incidence of GIST is increased, and multiple
GISTs are present in the small intestine ${ }^{[12]}$. Familial GIST was not detected in our series.

The symptoms usually vary depending on the size of the organs and tumor in which the GIST is located. GISTs smaller than $2 \mathrm{~cm}$ are usually asymptomatic, and they are detected incidentally during surgery, radiology, or endoscopy performed for other reasons. The most frequently seen symptom is gastrointestinal bleeding due to mucosal ulcerations [13]. Another frequent presentation is intraabdominal mass. In our series, a patient who underwent laparotomy due to intraabdominal mass was observed which originated jejunum mesentery and total excision was performed. In 4 cases, surgery was performed under urgent conditions due to gastrointestinal bleeding. Patients with esophageal tumors can present with dysphagia, odynophagia, chest pain, or hematemesis ${ }^{[14]}$. Gastric GIST cases can complain of nausea, vomiting, weight loss, and bloating. GISTs located in the small intestine may cause melena, obstruction, and perforation, whereas those with duodenal location may cause biliary obstruction ${ }^{[13,14]}$. They may present with acute abdominal findings when the tumor is ruptured ${ }^{[1]}$.

GISTs can often grow from the serosal surface to the abdominal cavity and sometimes into the lumen as a polypoid mass. For this reason, it reaches large sizes before it becomes symptomatic. In our series, a tumor that was detected was approximately $20 \times 20 \mathrm{~cm}$ in size (Fig. 3) [13].

Biopsy is mandatory for definitive diagnosis of GIST, but it is not recommended for surgery in tumors surgically removed due to risk of rupture of the fragile structure of the tumor and risk of hemorrhage due to its hypervascularity ${ }^{[4]}$.

Because of variable clinical presentations, any diagnostic protocol does not exist, and all endoscopic and imaging modalities can be used for the diagnosis and localization of the tumor. Endoscopic ultrasonography is the gold standard for diagnosis because it also provides the possibility of biopsy. Computed tomography (CT) is a useful technique for evaluating tumor size, determining local proliferation and

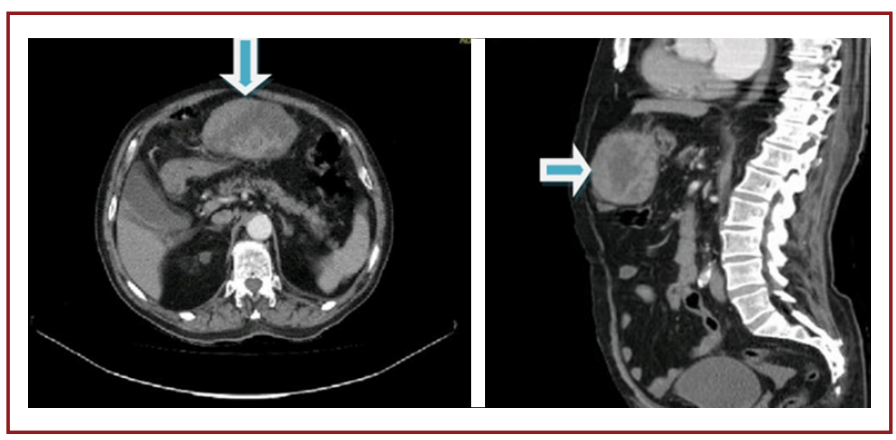

Figure 3. Computed tomographic image of gastric GIST. 
staging. Although positron emission tomography is a useful option in tumor staging, it is an objective test to evaluate the response in essentially imatinib-treated cases ${ }^{[14]}$. In our series, endoscopy and CT were used in the diagnosis (Figs. 3, 4). In the 2012 edition of the treatment guide prepared by the National Comprehensive Cancer Network in the United States, the surgeon's goal is defined as the complete removal of the target with a negative microscopic border, which includes the pseudocapsule around the tumor ${ }^{[1]}$. To achieve this, wedge-shaped or segmental resections $1 \mathrm{~cm}$ away from the macroscopic border are usually sufficient. In some cases, surrounding tissues or organs with tumor spread may need to be included in the resection [2].

During laparotomy, peritoneal surfaces, the rectovasical or rectovaginal space, bladder, and liver should be carefully explored for the presence of metastases. Encompassing operations may be required depending on the tumor location. A total gastrectomy for GISTs in the proximal stomach or gastroesophageal area, pancreaticoduodenectomy for the duodenal GIST (Whipple operation), and abdominoperineal resection (Miles operation) for the lower rectal tumors may be required ${ }^{[1]}$. Even though endoscopic resection of small stomach GISTs has been described, it is still not preferred due to a positive resection margin, tumor spread, and gastric perforation risks ${ }^{[15]}$. Since the lymph node involvement is rarely seen, routine lymphadenectomy is not recommended unless macroscopic involvement is observed ${ }^{[1,4,11]}$. In all our cases, resections could be applied with the achievement of negative surgical borders. In the patient who underwent abdominoperineal resection (Miles operation) due to mass in the lower rectum, metastasis was detected in one lymph node.

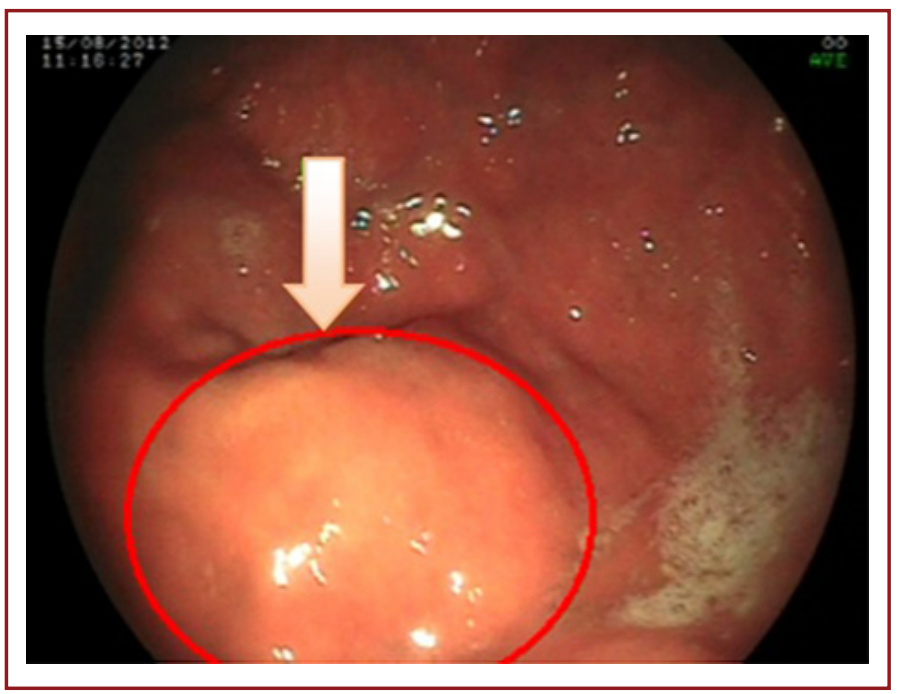

Figure 4. Endoscopic appearance of GIST on gastric antrum.

Laparoscopic resection has been shown to be safe and feasible in low-risk gastric GISTs $<5 \mathrm{~cm}$. In our series, a gastric GIST with a low risk was subjected to laparoscopic wedge resection.

Distant metastases are most common in the liver $(50 \%-$ $60 \%)$ and peritoneum (20\%-40\%), but rarely in the lung and bones (10\%). Rectal GIST is especially metastatic to the lungs ${ }^{[16]}$. Metastasis was not detected in our series.

The number of mitoses, tumor size, its origin, and type of resection are important in the prognosis. A disruption in the capsule integrity after the rupture of the tumor during resection, tumor necrosis, tumor size of $5 \mathrm{~cm}$, and more than 5 mitoses in the tumor under microscope at $50 \times \mathrm{mi}-$ croscopic magnification are associated with poor prognosis ${ }^{[4]}$. A significant cellularity, increased mitosis, large tumor diameter, and invasion of the mucosa are associated with malignancy. They are seen in $40 \%$ the cases with malignant intestinal GISTs, and $20 \%$ of cases with gastric GISTs. Gastric GISTs are more benign than small intestinal GISTs, while those localized in the esophagus, colon, or anorectal area have a more aggressive course ${ }^{[14]}$.

A practical staging system for GIST after surgical resection was reported by Bucher et al. ${ }^{[17]}$ (Table 1). It is based on five minor criteria (tumor size $\geq 5 \mathrm{~cm}$, mitotic index $\geq 5$ mitoses, the presence of necrosis, spread to the surrounding tissue, MIBI (Ki-67) index >10\%), and 2 major (presence of lymph node invasion or metastasis) criteria. Tumors having less than 4 minor criteria are classified as "low-grade GIST," and those with 4 or 5 minor criteria, or 1 major criteria as "highgrade GIST" [17]. In our series, 5 cases were in the high-risk group (Fig. 2). A 5-year survival rate after complete resection ranged between $34 \%$ and $60 \%{ }^{[11]}$.

Drug treatment should be preferred in patients with widespread metastases or those who cannot undergo a total excision due to local progression and patients whose general condition is not suitable for surgery. In the year 2002, the use of imatinib, a tyrosine kinase inhibitor in the CD117

Table 1. Surgical Staging According to Bucher et al. ${ }^{\text {[17] }}$

\section{Major criteria}

1. Metastasis

2. Lymph node invasion

\section{Minor criteria}

1. Tumor size $\geq 5 \mathrm{~cm}$

2. Mitotic index $\geq 5$ mitoses

3. Presence of necrosis

4. Invasion into surrounding tissue

5. MIBI (Ki-67) index $>10 \%$
1 major criterion (+): High-grade GIST

$<4$ minor criteria $(+)$ : Low-grade GIST 4 or 5 minor criteria (+): High-grade GIST 
(c-kit) positive nonresectable and/or metastatic malignant GISTs, was approved by the Food and Drug Administration. Adjuvant imatinib therapy is recommended in high-grade GIST patients. With imatinib, remission, and regression in $50 \%-80 \%$ of the cases can be achieved. In addition, imatinib is the first choice in neoadjuvant treatment, and in the treatment of residual tumor after surgery, recurrent, or metastatic GIST. Sunitinib is used for imatinib tolerance or failure ${ }^{[11]}$.

\section{Conclusion}

In conclusion, GIST is most frequently seen in the elderly population and especially in the stomach. Removal of the tumor with a clean surgical margin is the gold standard. The tumor size and the number of mitoses are the most important prognostic factors. In high-risk cases, imatinib is very useful in the treatment of recurrent or metastatic GISTs.

Peer-review: Externally peer-reviewed.

Authorship Contributions: Concept: O.A., T.A.; Design: O.A., T.A., T.T.E.; Data Collection or Processing:T.A., B.B.; Analysis or Interpretation: O.A., T.A., B.B., J.S.; Literature Search: T.A., J.S.; Writing: O.A., T.A., I.A.Ö., T.T.E.

Conflict of Interest: None declared.

Financial Disclosure: The authors declared that this study received no financial support.

\section{References}

1. Demetri GD, von Mehren M, Antonescu CR, DeMatteo RP, Ganjoo KN, Maki RG, et al. NCCN Task Force report: update on the management of patients with gastrointestinal stromal tumors. J Natl Compr Canc Netw 2010;8 Suppl 2:S1-41. [CrossRef]

2. Bilgen $K$, Hasçiçek SC, Çetinkünar S, Celep B, Tokgöz S, Tunç $\mathrm{G}$, et al. Atlı. Gastrointestinal Stromal Tümörlerde Prognostik Faktörler. Yeni Tıp Dergisi 2011;28:95-9.

3. Gupta P, Tewari M, Shukla HS. Gastrointestinal stromal tumor. Surg Oncol 2008;17:129-38. [CrossRef]

4. Kingham TP, DeMatteo RP. Multidisciplinary treatment of gastrointestinal stromal tumors. Surg Clin North Am 2009;89:217-33. [CrossRef]

5. Kindblom LG, Remotti HE, Aldenborg F, Meis-Kindblom JM. Gastrointestinal pacemaker cell tumor (GIPACT): gastroin- testinal stromal tumors show phenotypic characteristics of the interstitial cells of Cajal. Am J Pathol 1998;152:1259-69.

6. Blay JY, Bonvalot S, Casali P, Choi H, Debiec-Richter M, Dei Tos $A P$, et al.; GIST consensus meeting panelists. Consensus meeting for the management of gastrointestinal stromal tumors. Report of the GIST Consensus Conference of 20-21 March 2004, under the auspices of ESMO. Ann Oncol 2005;16:566-78.

7. Heinrich MC, Corless CL, Demetri GD, Blanke CD, von Mehren $M$, Joensuu $H$, et al. Kinase mutations and imatinib response in patients with metastatic gastrointestinal stromal tumor. J Clin Oncol 2003;21:4342-9. [CrossRef]

8. Pidhorecky I, Cheney RT, Kraybill WG, Gibbs JF. Gastrointestinal stromal tumors: current diagnosis, biologic behavior, and management. Ann Surg Oncol 2000;7:705-12. [CrossRef]

9. Sakamoto Y, Yamamoto J, Takahashi H, Kokudo N, Yamaguchi T, Muto T, et al. Segmental resection of the third portion of the duodenum for a gastrointestinal stromal tumor: a case report. Jpn J Clin Oncol 2003;33:364-6. [CrossRef]

10. Fletcher CD, Berman JJ, Corless C, Gorstein F, Lasota J, Longley $B J$, et al. Diagnosis of gastrointestinal stromal tumors: A consensus approach. Hum Pathol 2002;33:459-65. [CrossRef]

11. Folgado Alberto $S$, Sánchez $P$, Oliveira M, Cuesta L, Gomes F, Figueiredo A, et al. Gastrointestinal stromal tumors - a retrospective study of 43 cases. [Article in Spanish]. Rev Esp Enferm Dig 2008;100:696-700. [CrossRef]

12. Boldorini $R$, Tosoni $A$, Leutner $M$, Ribaldone $R$, Surico $N$, Comello $\mathrm{E}$, et al. Multiple small intestinal stromal tumours in a patient with previously unrecognised neurofibromatosis type 1: immunohistochemical and ultrastructural evaluation. Pathology 2001;33:390-5. [CrossRef]

13. van der Zwan SM, DeMatteo RP. Gastrointestinal stromal tumor: 5 years later. Cancer 2005;104:1781-8. [CrossRef]

14. Levy AD, Remotti HE, Thompson WM, Sobin LH, Miettinen M. Gastrointestinal stromal tumors: radiologic features with pathologic correlation. Radiographics 2003;23:283-304.

15. Piccinni G, Marzullo A, Angrisano A, lacobone D, Nacchiero $M$. Endoscopic resection of benign very low-risk gastric gastrointestinal stromal tumors. Is it enough? Eur J Gastroenterol Hepatol 2007;19:177-9. [CrossRef]

16. D'Amato G, Steinert DM, McAuliffe JC, Trent JC. Update on the biology and therapy of gastrointestinal stromal tumors. Cancer Control 2005;12:44-56. [CrossRef]

17. Bucher $P$, Egger JF, Gervaz $P$, Ris F, Weintraub D, Villiger $P$, et al. An audit of surgical management of gastrointestinal stromal tumours (GIST). Eur J Surg Oncol 2006;32:310-4. [CrossRef] 